\title{
Solving Geometric Constraint Systems II. A Symbolic Approach and Decision of Rc-constructibility ${ }^{1)}$
}

\author{
Xiao-Shan $\mathrm{Gao}^{2)}$ and Shang-Ching $\mathrm{Chou}^{3)}$
}

\begin{abstract}
This paper reports a geometric constraint solving approach based on symbolic computation. With this approach, we can compute robust numerical solutions for a set of equations. We give complete methods of deciding whether the constraints are independent and whether a constraint system is over-constraint. Also, over-constrainted systems can be handled naturally. Based on symbolic computation, we also give a decision procedure for the problem of deciding whether a constrainted diagram can be constructed with ruler and compass (rc-constructibility).
\end{abstract}

\section{Introduction}

In Part I of this paper [10], we use a construction approach to solve geometric constraint systems. More precisely, the algorithm tries to find a drawing procedure for a constrainted diagram with ruler and compass. The advantage of this approach and all the other construction approaches $[11,15,20]$ is that once a solution is found, the remaining steps of drawing this diagram becomes easy. Unfortunately, construction approaches are generally not complete, that is, the methods can not find drawing procedures for some of the diagrams that can be drawn with ruler and compass. In this paper, we study two complete approaches of solving geometric constraint systems: the symbolic approach and a decision method for ruler and compass constructibility based on symbolic computation.

The symbolic approach is quite similar to the numerical approach $[16,19]$. In the symbolic approach, we also first transform the geometric constraints into algebraic equations by assigning each point its coordinates. After that, instead of using numerical methods to solve the algebraic equation set, we will use general symbolic methods such as Ritt-Wu's decomposition algorithm [23, 25] or the Groebner basis method [1] to change the equation set to new forms which are easy to solve. Finally, we solve these new equations numerically. The most often used easy-solving equation form is the triangular form (see Section 2 for precise definition). The advantage of the symbolic approach is that once an equation set of triangular form is obtained, the solution of these equations is much easier than that of the equation set in general form. In some of the numerical approaches [16, 19], the equations are also simplified, while triangulation could be considered the ultimate simplification.

\footnotetext{
${ }^{1)}$ This work was supported in part by the NSF Grant CCR-9420857 and the Chinese National Science Foundation.

${ }^{2)}$ Institute of Systems Sciences, Academia Sinica, Beijing 100080. email:xgao\&mmrc.iss.ac.cn

${ }^{3)}$ Department of Computer Science, The Wichita State University Wichita KS 67260-0083, USA e-mail: choucs.twsu.edu
} 
In this paper, we use Ritt-Wu's decomposition algorithm [25, 23] to solve geometric constraint systems. We will discuss the problem of using special coordinates to simplify the triangular sets and how to transform the solutions of a triangular set with special coordinates to the general position.

Distinctive feature of this approach includes. (1) We can compute robust numerical solutions from an equation set of triangular form. (2) We give complete methods of deciding whether the constraints are independent and whether a constraint system is over-constraint. (3) Different from the construction approach [11, 15, 20, 10], both the numerical and the symbolic approaches can be used to solve constraint systems in $2 D$ and $3 D$ uniformly, since both approaches are capable of handling equations with any number of variables.

Our program based on Ritt-Wu's decomposition method is quite efficient. All of the 134 constraint systems reported in [10] can be solved within seconds. For more complicated problems like the regular pentagon problem (Example 2.1), the program can give a much clearer picture than the numerical approach and can lead to efficient and robust solutions.

Related to the symbolic approach, Hoffmann used the Grobner basis method to solve some special constraint systems [11]. Kondo used the Groebner basis method to test whether two dimensional constraints are independent, and if not, to find the relation between them [14]. In [4], Ritt-Wu's method and the Grobner basis method were used to find relations among any geometry quantities.

By transforming the constraint system into algebraic form, we can give a decision procedure for the problem of deciding whether a diagram can be constructed with ruler and compass (rc-constructibility). The problem of rc-constructibility is reduced to decide whether the roots of a univariate equation of degree $2^{k}(k>1)$ and with rational functions of parameters as coefficients can be solved in terms of square roots. Our decision procedure is quite efficient for the special case of quartic equation. Most geometric problems give equations of degree either two or four. For instance, $90 \%$ of the 134 problem reported in [10] give equations of degree one or two and all the remaining problems give equations of degree four.

In [20], Owen proved that his algorithm of solving constraint systems is complete if the constraint system uses only one predicate - distance between two points - and the distances used are algebraiclly independent. Of course, Owen's approach is not based on symbolic computation. It is still very desirable to develop a complete rc-constructibility method without using symbolic computations. A challenge problem is to find a set of construction rules which can be used to draw regular polygons with five or seventeen sides.

The rest of the paper is organized as follows. In Section 2., we describe how to solve constraint system with Ritt-Wu's decomposition algorithm. In Section 3., we give the decision procedure for the rc-constructibility.

\section{Solving Geometric Constraint Systems with Ritt-Wu's Decomposition Algo- rithm}

2.1. Basic Results of Ritt-Wu's Decomposition Algorithm. We only introduce the results needed in this paper. For more discussions about the method, see $[23,25,3]$. Let $\mathbf{Q}$ be the field of rational numbers, and $\mathbf{Q}\left[x_{1}, \ldots, x_{n}\right]$ or $\mathbf{Q}[X]$ the set of polynomials in variables $x_{1}, \ldots, x_{n}$. For $P \in \mathbf{Q}[X]-\mathbf{Q}$, we can write $P=c_{d} x_{p}^{d}+\ldots+c_{1} x_{p}+c_{0}$, where 
$c_{i} \in B\left[x_{1}, \ldots, x_{p-1}\right], p>0$, and $c_{d} \neq 0$. We call $p$ the class, $c_{d}$ the initial, and $x_{p}$ the leading variable of $P$, or class $(P)=p$, init $(P)=c_{d}$, and $l v(P)=x_{p}$.

A sequence of polynomials $A S C=A_{1}, \ldots, A_{p}$ in $\mathbf{Q}[X]$ is said to be in triangular form, if either $p=1$ and $A_{1} \neq 0$ or $0<\operatorname{class}\left(A_{i}\right)<\operatorname{class}\left(A_{j}\right)$ for $1 \leq i<j$.

Let $P S$ be a polynomial set in $\mathbf{Q}[X]$. For an extension field $E$ of $\mathbf{Q}$, like the complex number field, let

$$
Z e r o(P S)=\left\{a \in E^{n} \mid \forall P \in P S, P(a)=0\right\}
$$

For a polynomial set $P S$ and a polynomial $D$ in $\mathbf{Q}[X]$, we define

$$
Z \operatorname{ero}(P S / D)=Z \operatorname{ero}(P S)-Z \operatorname{ero}(D) \text {. }
$$

For a polynomial set $P S$ in $\mathbf{Q}[X]$, Ritt-Wu's zero decomposition algorithm can be used to represent the zero set of $P S$ as the union of the zero sets of polynomial sets in triangular form. More precisely, we have [25, 3]

$$
\operatorname{Zero}(P S)=\bigcup_{1 \leq i \leq k} \operatorname{Zero}\left(T S_{i} / I_{i}\right)
$$

where each $T S_{i}$ is a polynomial set in triangular form and $I_{i}$ is the production of the initials of the polynomials in $T S_{i}$.

Let $T S=\left\{P_{1}, \ldots, P_{p}\right\}$ be a polynomial set in triangular form. We rename the leading variables of $P_{i}$ as $y_{i}$ and the remaining variables as $u_{1}, \ldots, u_{q}(p+q=n)$. Then $T S$ can be written as

$$
P_{1}\left(U, y_{1}\right), P_{2}\left(U, y_{1}, y_{2}\right), \cdots, P_{p}\left(U, y_{1}, y_{2}, \ldots, y_{p}\right)
$$

where $U=u_{1}, \ldots, u_{q}$.

In Section 3., we need the following result $[23,9]$. For each triangular set $T S$ of the form (2.2), let $I$ be the production of the initials of its polynomials. Then we can find a new variable $w$ such that $Z \operatorname{ero}(T S / I)$ can be obtained as follows.

$$
R(U, w)=0, y_{i}=M_{i}(U, w) / N_{i}(U, w),(i=1, \ldots, p)
$$

where $R, M_{i}$, and $N_{i}$ are polynomials in $\mathbf{Q}[U, w]$. In other words, the solutions of a polynomial set in triangular form can be reduced to the roots of a univariate polynomial equation.

The reason we use triangular form is that the solution of a set of polynomial equations in triangular form is easy to compute. Take $T S$ in (2.2) as an example. For a set of numerical values for the $u_{i}, P_{1}=0$ becomes a univariate equation. We can find the solutions for $y_{1}$ from this univariate equation. Next, by substituting the numerical solution for $y_{1}$ into equation $P_{2}, P_{2}=0$ becomes a univariate equation. We now solve $y_{2}$ from this univariate equation, and so on. According to (2.1), we need to find the zero set $\operatorname{Zero}\left(T S_{i} / I_{i}\right)$. Since $I_{i} \neq 0$, we can discard any solutions that vanishes $I_{i}$. Thus for each set of values for the $u_{i}$, either $\operatorname{Zero}\left(T S_{i} / I_{i}\right)$ is empty or we can find solutions for all the $y_{i}$ according to the above procedure.

2.2. A Non-trivial Example. Let us first give an example. We will use this example in the following sections whenever it is needed. 
Example 2.1 (Regular Pentagon) Draw a regular pentagon ABCDE whose edge is $r$.

We first assign the following coordinates to the five points: $A=(0,0), B=(r, 0), C=$ $\left(x_{2}, y_{2}\right), D=\left(x_{3}, y_{3}\right), E=\left(x_{4}, y_{4}\right)$. Since there are five points in the diagram, we need $2 * 5-3=7$ constraints to determine the shape of the diagram. The constraint $|A B|=r$ is already satisfied automatically. We need six other constraints to determine the six variables $x_{2}, y_{2}, x_{3}, y_{3}, x_{4}, y_{4}$. The following are these constraints and their corresponding algebraic equations.

$$
\begin{array}{ll}
|A B|=|A E|: & h_{1}=y_{4}^{2}+x_{4}^{2}-r^{2}=0 \\
|A B|=|B C|: & h_{2}=y_{2}^{2}+x_{2}^{2}-2 r x_{2}=0 \\
|A B|=|C D|: & h_{3}=y_{3}^{2}-2 y_{2} y_{3}+x_{3}^{2}-2 x_{2} x_{3}+y_{2}^{2}+x_{2}^{2}-r^{2}=0 \\
|A B|=|D E|: & h_{4}=y_{4}^{2}-2 y_{3} y_{4}+x_{4}^{2}-2 x_{3} x_{4}+y_{3}^{2}+x_{3}^{2}-r^{2}=0 \\
|A D|=|D B|: & h_{5}=2 r x_{3}-r^{2}=0 \\
|A C|=|C E|: & h_{6}=y_{4}^{2}-2 y_{2} y_{4}+x_{4}^{2}-2 x_{2} x_{4}=0
\end{array}
$$

Using Rit-Wu's decomposition algorithm, we have

$$
\operatorname{Zero}\left(\left\{h_{1}, \ldots, h_{6}\right\}\right)=\cup_{i=1}^{4} Z \operatorname{ero}\left(T S_{i} / I_{i}\right)
$$

where $T S_{i}$ are given below.

$$
\begin{array}{llll}
T S_{1}= & T S_{2}= & T S_{3}= & T S_{4}= \\
y_{4}-y_{2} & r y_{4}+\left(2 x_{2}-2 r\right) y_{2} & y_{4}-y_{2} & y_{4} \\
x_{4}+x_{2}-r & 2 x_{4}-2 x_{2}+r & x_{4}+x_{2}-r & x_{4}+r \\
r y_{3}+\left(-2 x_{2}+r\right) y_{2} & r y_{3}+\left(2 x_{2}-3 r\right) y_{2} & r y_{3}+\left(2 x_{2}-3 r\right) y_{2} & y_{3}-y_{2} \\
2 x_{3}-r & 2 x_{3}-r & 2 x_{3}-r & 2 x_{3}-r \\
4 y_{2}^{2}-2 r x_{2}-r^{2} & 4 y_{2}^{2}-2 r x_{2}-r^{2} & 4 y_{2}^{2}-2 r x_{2}-r^{2} & 4 y_{2}^{2}+5 r^{2} \\
4 x_{2}^{2}-6 r x_{2}+r^{2} & 4 x_{2}^{2}-6 r x_{2}+r^{2} & 4 x_{2}^{2}-6 r x_{2}+r^{2} & 2 x_{2}+r
\end{array}
$$

$T S_{4}$ does not have non-zero real zeros, since there is a polynomial $4 y_{2}^{2}+5 r^{2}$. Each of $T S_{1}, T S_{2}$, and $T S_{3}$ contains six polynomials and has total degree four, i.e., for a non-zero value of $r$, each of $T S_{1}, T S_{2}$, and $T S_{3}$ gives four solutions to the problem (see Figures 1 and 2).

2.3. Coordinate Transformation. In this section, we will discuss the problem of how to draw the constrainted diagram on a window system after a triangular decomposition like (2.1) has been given.

To improve the efficiency of Ritt-Wu's decomposition algorithm, we always use special coordinates for some points. In the pentagon example, points $A=(0,0), B=(r, 0)$ have special coordinates. This example actually gives some insight into the general case. If a plane geometric constraint system involves $n$ points, we generally need $2 n-3$ independent (i.e., no redundancies) constraints to determine the shape of the corresponding diagram. Such a constraint system or its corresponding diagram is called well-defined. There are three free parameters in a well-defined diagram: the coordinates of a point $P_{0}$ and the direction of a line $P_{0} P_{1}$. When using Ritt-Wu's decomposition algorithm, we always assume that the coordinates of $P_{0}$ are $(0,0)$ and the coordinates of $P_{1}$ are $(r, 0)$ where $r$ is a variable representing the length of $P_{0} P_{1}$. This will make the decomposition much faster.

When drawing the constrainted diagram on a window, coordinates of point $A$ are usually not $(0,0)$ and line $P_{0} P_{1}$ is usually not horizontal. We thus need to do certain coordinate 
transforms before we can use the triangular equations to find the coordinates of a diagram in the general position. Before we can do that, let us consider three basic transformations for a well-defined diagram. For a well-defined constraint system, let $P_{0}$ be the point with free position and $P_{0} P_{1}$ the line with free direction. Then we usually can perform the following transformations.

1. Translate point $P_{0}$.

2. Rotate line $P_{0} P_{1}$ around point $P_{0}$ with the length of $P_{0} P_{1}$ fixed.

3. If the length of a segment or the value of an angle is given as a parameter, we can change the segment length or the angle value.

The coordinate transformations for the three transformations are well known. We will show that in our case what modifications can be made. For case (1), let the original and the new coordinates of point $P_{0}$ be $\left(x_{0}, y_{0}\right)$ and $\left(x_{0}^{\prime}, y_{0}^{\prime}\right)$. The new coordinates for other points can be obtained with the following transformation

$$
x^{\prime}=x+\left(x_{0}^{\prime}-x_{0}\right), y^{\prime}=y+\left(x_{0}^{\prime}-x_{0}\right) .
$$

For case (2), let the old coordinates of $P_{0}$ and $P_{1}$ be $\left(x_{0}, y_{0}\right)$ and $\left(x_{1}, y_{1}\right)$. Suppose, the new direction of $P_{0} P_{1}$ is determined by $P_{0} Q$ where $Q$ is a new point $Q=\left(x_{2}, y_{2}\right)$. The new coordinates for all points can be obtained with the following transformation

$$
x^{\prime}=x_{0}+\cos \theta *\left(x-x_{0}\right)+\sin \theta *\left(y-y_{0}\right), y^{\prime}=y_{0}-\sin \theta *\left(x-x_{0}\right)+\cos \theta *\left(y-y_{0}\right)
$$

where $\theta=\arctan \left(\frac{\left(y_{2}-y_{0}\right)\left(x_{1}-x_{0}\right)-\left(y_{1}-y_{0}\right)\left(x_{2}-x_{0}\right)}{\left(x_{1}-x_{0}\right)\left(x_{2}-x_{0}\right)+\left(y_{2}-y_{0}\right)\left(y_{1}-y_{0}\right)}\right)$ is between $-\pi / 2$ and $\pi / 2$. To avoid the computation of trigonometric functions, the new position of point $P_{1}$ can be obtained as the intersection of the line $P_{0} Q$ and the circle with center $P_{0}$ and radius $P_{0} P_{1}$. Of the two intersections, we choose the one which is near $P_{1}$. For case $(3)$, since the parameters are in the equations explicitly, the solution is straightforward.

Now we can give the procedure of computing the coordinates of the points in a diagram.

1. For a given set of numerical values for the segment lengths and angles, we can use the original triangular set $T S$ to obtain a set of solutions for the coordinates of the points.

2. For each given new position of $P_{0}$ and a new direction of line $P_{0} P_{1}$, we can use transformations (2.5) and (2.6) to find the new coordinates for all other points.

For the complexity of the above procedure, the second step is clearly linear in the number of involving coordinates or the number of points in the diagram. In the first step, we need to solve a fixed number of univariate polynomial equations. We can use Newton's iteration method to do that [7]. To find all the real roots of a univariate equation, we can first use the algorithm reported in [5] to separate the roots of the equations and then use Newton's method to compute each of the root. More discussions about numerical methods of solving constraint systems can be found in $[16,19]$.

Comparing to the previous approaches, our approach has clear advantages. First, instead of solving equations in general form, we need only solve univariate equations due to the fact 
that the equations are in triangular form. As a consequence, we can find all the solutions if needed. This makes the selection of the so-called "robust" solutions possible. Second, for each set of segment lengths and angle values, we need only to solve a fixed number of univariate equations for one time. After a set of solutions is obtained, for each new position of $P_{0}$ and each new direction of $P_{0} P_{1}$ we need only to do a linear transformation to obtain their positions. Of course, in our approach, we need first to find a triangular decomposition, which might be time consuming. Fortunately, we need only to do that once for each constraint system. So we may adopt the following strategy: if in a fixed period of time, say five minutes, the program still cannot find the triangular decomposition then we will abandon the process and use the general numerical approach.

2.4. Over-Constrainted Systems. In many papers including Part I of this paper [11, $20,10]$, a constraint system is called well-defined if it has $n$ characteristic points and lines and $2 * n-3$ independent constraints. As we will show below, though good enough for the construction and AI approach to constraint solving, this definition does not give the whole picture. Generally, let us define a constraint system to be well-defined if the shape of the corresponding diagram has only a finite number of cases. We will show later that a well-defined constraint system of $n$ points may have more than $2 * n-3$ constraints.

Using algebraic languages, we can give a criteria for well-defineness. From the discussion in the last paragraph in Section 2.1, the following result is easy to prove.

Theorem 2.2 Let $P_{0}$ and $P_{1}$ be any two points in the constraint system such that length of $P_{0} P_{1}$ is $r$. Let $P_{0}=(0,0), P_{1}=(r, 0)$. Then the constraint system with $n$ points is welldefined iff in a decomposition like (2.1) each triangular set TS contains $2 n-4$ polynomials.

We use the pentagon example to show that sometimes we need more than $2 n-3$ constraints to define a constraint system with $n$ points. The following table gives the result of checking three more geometric conditions on the three diagrams defined by $T S_{1}, T S_{2}$, and $T S_{3}$ using the automated theorem proving methods given in $[25,3]$.

\begin{tabular}{l|ccc} 
& $T S_{1}$ & $T S_{2}$ & $T S_{3}$ \\
\hline$|A D|=|A C|$ & true & false & false \\
$|B D|=|B E|$ & true & true & false \\
$|E B|=|E C|$ & true & false & true \\
\hline
\end{tabular}

By Theorem 2.2, the pentagon problem in Section 2.2 is well-defined. But the seven independent constraints do not properly define a pentagon. From the above table, we see that only $T S_{1}$ defines a pentagon (Figure 1a). The solutions for $T S_{2}$ and $T S_{3}$ are not pentagons (Figures 1b and 1c). To define a proper pentagon, we need to add at least another constraint 


$$
|A D|=|A C|: \quad h_{7}=y_{3}^{2}+x_{3}^{2}-y_{2}^{2}-x_{2}^{2} .
$$

Now there is only one useful triangular set $T S_{1}$ in the decomposition:

$$
\operatorname{Zero}\left(\left\{h_{1}, \ldots, h_{7}\right\}\right)=\operatorname{Zero}\left(T S_{1} / I_{1}\right) \cup \operatorname{Zero}\left(T S_{4} / I_{4}\right) .
$$

But this constraint system is over-constraint in the sense that there are five points but eight constraints. Note that the last constrain $h_{7}=0$ does not reduce the dimension of freedom of the problem; it only eliminates eight of the twelve solutions of the original problem.

We have observed that for some constraint systems, we must use more than $2 n-3$ consistent constraints to properly determine the shape of the corresponding diagram. It is difficult for iteration numerical methods to deal with this kind of problems [16]. In the symbolic approach, this kind of "over-constraint" can be solved naturally. Furthermore, the triangular set of equations generally becomes simpler when more consistent constraints are added.

2.5. Robustness of Numerical Solving. It is well known (see e.g., [16]) that the Newton iteration method is unreliable, especially when the number of constraints are larger than the number of variables. From the symbolic viewpoint, this unreliability can be divided into two cases. (1) The first case occurs when a triangular set TS has more than one solutions. When changing the shape of the diagram continuously, the solver may "jump" from one branch of solutions to another branch. For instance, $T S_{1}$ for the pentagon has four branches of solutions. The two solutions given from the quadratic equation about $y_{2}$ are easy to understand: if $P$ is a solution pentagon then the reflection of $P$ with the $x$-axis, obtained by changing $y_{i}$ to $-y_{i}$, is also a solution. Now we need only to consider the two solutions given by the quadratic equation about $x_{2}$. The two solutions are two regular pentagons, one with $r$ as an edge another with $r$ as a diagonal (Figure 2).

In our case, this problem can be solved due to the way of solving equations. As we mentioned in the preceding section, for each set of segment length and angle values, we only solve a fixed number of non-linear univariate equations once and the coordinates for other new positions are given by linear transformations.

Another method is to reduce the branches of solutions by introducing more constraints. For the pentagon example, we can assume that $A B C D E$ is a concave pentagon by introducing a condition $\theta_{1}=\left(|A D|^{2}>|A B|^{2}\right)$ which means that the diagonal $A D$ is longer than the edge $A B$. When a set of solutions are found, we check if $\theta_{1}$ is true. If it is not true, we discard it. Similarly, we can assume that $A-B-C$ is in counterclockwise direction by introducing anther condition $\theta_{2}=\left(y_{2}>0\right)$. Now the pentagon problem has a unique solution and the solution will always be robust. In summary, the numerical method works as follows. Let us assume that the current value for a variable $x$ is $x_{0}$. When we drag a diagram, the next solution for $x$ is near $x_{0}$. Thus, we can use $x_{0}$ as an initial value to find the new value for $x$. We then check whether the constraints $\theta_{1}$ and $\theta_{2}$ are valid for these 
new values. If they are valid, we have found a set of solution. Otherwise, most probably we are near a "critical point" where more than one branches of solutions meet. Now we need to find all the solutions and to select one satisfying $\theta_{1}$ and $\theta_{2}$.

(2) The second case occurs if the problem is reducible, i.e., its solutions can be written as the union of the solutions of more than one triangular equation sets. If $h_{7}=0$ is not added, the pentagon problem has three group of solutions, and hence is reducible. In a reducible problem, when changing the shape of the diagram continuously, the solver may "jump" from the solutions of one triangular set to that of another triangular set. This problem can be solved trivially if a triangular decomposition is already given: different solutions are given by different triangular sets and will not be mixed.

2.6. Independent and Conflict Constraints. Recall that a set of constraints are said to be independent if no one of them can be removed and the new constraint system still defines the same diagram. A set of constraints are said to be conflict if no diagram in the Euclidean plane satisfying this constraint system.

Many results about the independence and conflict of constraints are reported in [21, 24]. One of the interesting result is as follows. If the only type of constraints is distance between two points and all the distances do not have algebraic relations, a constraint system is independent and non-conflict ${ }^{4)}$ if in any subsystem of $n$ points, the number of constraints $e \leq 2 n-3$. This and other results reported in [21], though important, have the following limitation. The distances and angles have to be algebraic independent which means that we can not deal with problems involving two points on the same circle or regular polygons let along constraint systems using the implicit constraints like "two segment are equal" and "two angles are equal". On the other hand, the symbolic approach can provide complete solution to the problem of independency and conflict in the general case.

Let us first comment that in the general case there is no connection between the concept of independency and conflict and the critical number $2 n-3$. Actually, there are conflict and/or non-independent systems which have less than $2 n-3$ constraints, as well as nonconflict and/or independent systems with more than $2 n-3$ constraints. For instance, the regular pentagon is a non-conflict and independent system with more than $2 n-3$ constraints. Also, almost every geometry theorem provides an example for non-independent systems with less than $2 n-3$ constraints.

2.6.1. Decision of Conflict Constraints. For a constraint system $C S$, let $P S$ be the corresponding algebraic equations of the constraints. The conflict of $C S$ means that $P S$ does not have solutions. In the case of complex number field, this can be decided with the following result. For the case of real numbers, a complete algorithm is provided by the quantifier elimination algorithm [6].

Theorem 2.3 ([25]) A set of polynomial equations $P S$ does not have solutions over the field of complex numbers iff $k=0$ in decomposition ${ }^{5)}$ (2.1).

If $k=0$ in decomposition (2.1), the constraint system is also conflict over the field of real numbers.

\footnotetext{
${ }^{4)}$ Here the concept of conflict is over the complex numbers. That is, a constraint system is non-conflict if there is a diagram over the field of complex numbers satisfying all the constraints.

${ }^{5)}$ Here we assume that the triangular sets are irreducible. For the definition of irreducible triangular sets, see $[23,25]$.
} 
In the pentagon example, if we want to draw a regular pentagon with edge 1 and diagonal 2 , we will get a conflict constraint system. In other words, $\operatorname{Zero}(P S)=\emptyset$ where $P S$ consists of equations for $|A B|=|B C|=|C D|=|D E|=|E A|=1$ and $|A D|=|D B|=|B E|=|E C|=$ 2 .

If two dimensional constrains are conflict, we can find the relation between the two dimensions. In [14], this is done with the Groebner basis. In [4], both the Groebner basis and Ritt-Wu's method are used to find such relations. In the regular pentagon problem, we just showed that the edge $r$ and the diagonal $d$ are two conflict dimensions. To find a relation between them, let $P S$ be the set of equations for the constraints $|A B|=|B C|=|C D|=$ $|D E|=|E A|=r$ and $|A D|=|D B|=|B E|=|E C|=d$. Using Ritt-Wu's decomposition algorithm, we have

$$
Z \operatorname{ero}(P S)=Z \operatorname{ero}\left(T S_{5} / I_{5}\right) \cup Z \operatorname{ero}\left(T S_{6} / I_{6}\right)
$$

where $T S_{5}$ and $T S_{6}$ are polynomial sets in triangular form and $I_{5}$ and $I_{6}$ are the product of the corresponding initials. The first polynomials in $T S_{5}$ and $T S_{6}$ are

$$
d^{2}+r d-r^{2}=0 \text { and } d^{2}-r d-r^{2}=0 .
$$

Since $d$ and $r$ are positive, the two reasonable solutions are $d=\frac{1+\sqrt{5}}{2} r$ and $d=\frac{-1+\sqrt{5}}{2} r$. The first one represents the case that $r$ is the edge and $d$ is the diagonal. The second one represents the case that $r$ is the diagonal and $d$ is the edge.

2.6.2. Decision of Independency. Let $P S$ be the equation set of a constraint system. This system is not independent iff there is a polynomial $P$ in $P S$ such that

$$
\operatorname{Zero}(P S-\{P\}) \subset \operatorname{Zero}(P) \text { or } \operatorname{Zero}(P S-\{P\})=\operatorname{Zero}(P S) \text {. }
$$

Theorem 2.4 If $|P S| \leq 2 n-3$, the constraints are independent iff in decomposition (2.1) there is at least one irredundant triangular set T $S_{i}$ containing $|P S|$ polynomials.

To prove this theorem, we need a theorem from algebraic geometry which says that the triangular set representing an irredundant component of $Z \operatorname{ero}(P S)$ cannot contain more than $|P S|$ polynomials [8]. If the constraints are not independent, $\operatorname{Zero}(P S)=\operatorname{Zero}(P S-$ $\{P\})$. According to above theorem, an irredundant triangular set contains less than $|P S|$ polynomials. This is contradict to the hypothesis of the theorem.

In the general case, we need to check whether there is a polynomial in $P S$ such that (2.8) is valid. Note that to check $(2.8)$ is actually to prove a geometry theorem. Let $C_{P}$ be the geometric constraint of $P=0$ and $C_{1}, \ldots, C_{n}$ the geometric constraints for other equations in $P S$. Then (2.8) is equivalent to the following geometry theorem

$$
\forall \text { points }\left[\left(C_{1} \wedge C_{2} \cdots \wedge C_{n}\right) \Rightarrow C_{P}\right]
$$

The problem of automated geometry theorem proving has been studies extensively [25, 3]. We may use these efficient algorithms to prove whether (2.8) is true.

Let us use the pentagon problem to show how Ritt-Wu's method is used to check the independency. Applying Theorem 2.4 to (2.4), we know that the seven constrains given in Section 2.2 are independent. Since adding constraint $|A D|=|A C|$ eliminates two components from (2.5) (see (2.7)), this new constraint is also independent to the seven constraints. Let us check 
whether a new constraint $|B D|=|B E|$ (with equation $h_{8}=y_{3}^{2}+x_{3}^{2}-2 r x_{3}-y_{2}^{2}-x_{2}^{2}+2 r x_{2}$ ) is independent to the eight constraints in (2.7). Equivalently, we want to know whether the new constraint is valid on $\operatorname{Zero}\left(\left\{h_{1}, \ldots, h_{7}\right\}\right)=\operatorname{Zero}\left(T S_{1} / I_{1}\right) \cup \operatorname{Zero}\left(T S_{4} / I_{4}\right)$. This can be done by computing the remainder of the pseudo division of polynomial $h_{8}$ with respect to $T S_{1}[23,25]$. This remainder is zero iff $h_{8}=0$ is valid on $Z \operatorname{ero}\left(T S_{1} / I_{1}\right)$. Following this procedure, we can show that $h_{8}=0$ is valid on $\operatorname{Zero}\left(T S_{1} / I_{1}\right)$ but not on $\operatorname{Zero}\left(T S_{4} / I_{4}\right)$. Since $T S_{4}$ does not has solutions in the field of real zeros, $h_{8}=0$ is not independent to $h_{1}=0, \ldots, h_{7}=0$ over the field of real number.

\section{A Decision Procedure for Rc-constructibility}

3..1. Basic Results on Rc-constructions. Let $\mathbf{Q}$ be the field of rational numbers. Using the symbolic approach mentioned before, solving a geometric constraint system can be reduced to solving a set of algebraic equations. We thus allow us to abuse terms by saying that the numerical solutions of the equations can be constructed with ruler and compass if the corresponding diagram can be constructed with ruler and compass.

It is well-known [13] that a number $\eta$ can be constructed with ruler and compass iff there exist $n$ real numbers $\eta_{1}, \eta_{2}, \ldots, \eta_{n}=\eta$ and $n$ quadratic equations

$$
\begin{aligned}
& Q_{1}\left(x_{1}\right)=x_{1}^{2}+b_{1} x_{1}+c_{1}=0 \\
& Q_{2}\left(x_{1} x_{2}\right)=x_{2}^{2}+b_{2}\left(x_{1}\right) x_{2}+c_{2}\left(x_{1}\right)=0 \\
& \ldots \\
& Q_{n}\left(x_{1}, x_{2}, \ldots, x_{n}\right)=x_{n}^{2}+b_{n}\left(x_{1}, \ldots, x_{n-1}\right) x_{n}+c_{n}\left(x_{1}, \ldots, x_{n-1}\right)=0
\end{aligned}
$$

such that $Q_{i}\left(\eta_{1}, \eta_{2}, \ldots, \eta_{i}\right)=0,(i=1, \ldots, n)$. Since $Q_{i}$ is of degree two, we can further assume that the $b_{i}$ and $c_{i}$ are linear in the variables $x_{1}, \ldots, x_{i-1}$. We assume that $x_{n-1}$ occurs in $Q_{n}$. Otherwise, $\eta_{n-1}$ is not needed to define $\eta$. Since $Q_{n}$ is linear in $x_{n-1}$, from $Q_{n}\left(\eta_{1}, \eta_{2}, \ldots, \eta_{n}\right)=0$, we have

$$
\eta_{n-1}=\frac{\eta_{n}^{2}+e_{n-1}\left(\eta_{1}, \ldots, \eta_{n-2}\right) \eta_{n}+f_{n-1}\left(\eta_{1}, \ldots, \eta_{n-2}\right)}{m_{n-1}\left(\eta_{1}, \ldots, \eta_{n-2}\right) \eta_{n}+n_{n-1}\left(\eta_{1}, \ldots, \eta_{n-2}\right)} .
$$

Substituting $\eta_{n-1}$ into $Q_{n-1}$ and taking the numerator, we have

$$
\eta_{n}^{4}+g_{3} \eta_{n}^{3}+g_{2} \eta_{n}^{2}+g_{1} \eta_{1}+g_{0}=0
$$

where $g_{i}$ are polynomials of $\eta_{1}, \ldots, \eta_{n-2}$. Since $Q_{i}$ is of degree two in $\eta_{i}$, we can assume that $g_{i}$ are linear in $\eta_{1}, \ldots, \eta_{n-2}$. Then

$$
\eta_{n-2}=\frac{\eta_{n}^{4}+P_{n-2}\left(\eta_{1}, \ldots, \eta_{n-3}, \eta_{n}\right)}{Q_{n-2}\left(\eta_{1}, \ldots, \eta_{n-3}, \eta_{n}\right)}
$$

where $P_{n-2}$ and $Q_{n-2}$ are polynomials with degree three in $\eta_{n}$. Repeat this process, we have

$$
\eta_{1}=\frac{\eta_{n}^{2^{n-1}}+P_{1}\left(\eta_{n}\right)}{Q_{1}\left(\eta_{n}\right)}
$$

where $P_{1}$ and $Q_{1}$ are polynomials of degree $2^{n-1}-1$. Substituting (3.1) into $Q_{1}\left(\eta_{1}\right)=$ $\eta_{1}^{2}+b_{1} \eta_{1}+c_{1}=0$ and taking the numerator, we have

$$
\eta_{n}^{2^{n}}+h_{2^{n}-1} \eta_{n}^{2^{n}-1}+\ldots+h_{0}=0
$$


As a consequence, we have the well known result: an rc-constructible number is the root of an equation of degree $2^{k}$ for some $k>0$. Therefore, if the roots of an irreducible polynomial equation $P(x)=0(P(x)) \in \mathbf{Q}[x])$ can be constructed by ruler and compass, its degree must be a power of two. Thus the root of a cubic polynomial equation is rc-constructible iff the polynomial is reducible.

Since we can efficiently factor polynomials with computer algebraic systems such as Maple [2], the only remaining problem is to decide whether the roots of polynomial equations of degree $2^{k}$ are rc-constructible.

3..2. Quartic Equations. Let us first consider a special case: we will give an efficient method of deciding whether the roots of a quartic equation are rc-constructible. Let a quartic equation in $K[x]$ be

$$
x^{4}+h_{3} x^{3}+h_{2} x^{2}+h_{1} x+h_{0}=0
$$

where $K$ is field. In our case, $K$ could be the field of rational numbers $\mathbf{Q}$ or the field of rational functions $\mathbf{Q}\left(u_{1}, \ldots, u_{m}\right)$. If $K=\mathbf{Q}\left(u_{1}, \ldots, u_{m}\right)$, we say that the roots of the equation (3.3) are rc-constructible if for all possible rational numbers $r_{1}, \ldots, r_{m}$ the roots of the quartic equation obtained by substituting $r_{i}$ for $u_{i}$ are rc-constructible.

Let us assume that the quartic polynomial in (3.3) is irreducible. If a root $y$ of (3.3) is rc-constructible, $y$ can be written as the solution of the following two equations

$$
\begin{aligned}
& x_{1}^{2}+b x_{1}+c=0 \\
& y^{2}+\left(-m x_{1}+f\right) y-n x_{1}+g=0
\end{aligned}
$$

The first equation can be simplified to $x_{1}^{2}+d=0$. Since the roots are real numbers, we can further assume that the first equation is $x_{1}^{2}-1=0$. From the second equation we have $x_{1}=\frac{y^{2}+f y+g}{m y+n}$. Substitute this into $x_{1}^{2}-1=0$, the numerator is

$$
\left(y^{2}+f y+g\right)^{2}-(m y+n)^{2}=0
$$

Comparing the coefficients of this quartic equation with that of (3.3), we obtain a set of equations

$$
\begin{aligned}
& 2 f-h_{3}=0 \\
& m^{2}-2 g-f^{2}+h_{2}=0 \\
& 2 m n-2 f g+h_{1}=0 \\
& n^{2}-g^{2}+h_{0}=0
\end{aligned}
$$

Eliminating $f, m$, and $n$, we have

$$
8 g^{3}-4 h_{2} g^{2}+\left(2 h_{1} h_{3}-8 h_{0}\right) g-h_{0} h_{3}^{2}+4 h_{0} h_{2}-h_{1}^{2}=0
$$

Let $g_{1}$ be a root of equation (3.4), $f_{1}, m_{1}$ and $n_{1}$ solutions for $f, m$ and $n$ with $g$ substituted by $g_{1}$. Then all the roots of equation (3.3) can be obtained from the quadratic equations

$$
y^{2}+f_{1} y+g_{1} \pm\left(m_{1} y+n_{1}\right)=0 .
$$

Therefore, the roots of equation (3.3) can be obtained by successively solving several quadratic and a cubic equations. By the discussion in the end of Section 3..1, roots of a cubic equation 
are rc-constructible iff the cubic polynomial is reducible, i.e., it has a linear factor. Then we have a method of deciding whether the roots of a quartic equation can be constructed with ruler and compass.

Since $2 m n=2 f g-h_{1}$ is a constant for fixed values of $f$ and $g$, for each nonzero value of $m$ there is a unique value for $n$. Furthermore, if $m$ changes signs, $n$ also changes its sign. From this observation, one of the two quadratic equations, say $\left(a x^{2}+2 b x+c+2 a o\right)-(2 m x+n)=0$ is enough to obtain all the roots of equation (3.3) if we take the positive and negative square roots for $m$ or $n$. Here is a summary of what we have proved.

Theorem 3.1 (1) Use the notations given above. The roots of equation (3.3) are rcconstructible iff the cubic polynomial in (3.4) has a linear factor over the field $K$.

(2) Let $g-g_{0}$ be a linear factor of the polynomial in (3.4). Then the four roots of equation (3.3) are given by the following triangular set of linear and quadratic equations

$$
\begin{aligned}
& y^{2}+f y+g-(m y+n)=0 \\
& 2 m n-2 f g+h_{1}=0 \\
& m^{2}-2 g-f^{2}+h_{2}=0\left(\text { or } n^{2}-g^{2}+h_{0}=0 \text { if } m=0\right) \\
& 2 f-h_{3}=0 \\
& g-g_{0}=0
\end{aligned}
$$

Example 3.2 (Pappus' Problem I) Given a fixed point $P$, a fixed angle $A O B$, and a fixed length $d$, draw a line passing through point $P$ and cutting lines $O A$ and $O B$ with a segment of length $d$.

Let the segment be $X Y$. Use the following coordinates $O=(0,0), A=(w, 0), P=(u, v), B=$ $(w, s), X=\left(x_{1}, y_{1}\right), Y=\left(x_{2}, 0\right)$. The algebraic equations of the constraints are

$$
\begin{array}{lr}
f_{1}=w y_{1}-s x_{1}=0 & X, O, B \text { are collinear } \\
f_{2}=\left(y_{1}-v\right) x_{2}-u y_{1}+v x_{1}=0 & X, P, Y \text { are collinear } \\
f_{3}=x_{2}^{2}-2 x_{1} x_{2}+y_{1}^{2}+x_{1}^{2}-d_{1}^{2}=0 & |X Y|=d_{1}
\end{array}
$$

If considering $w, u, v$, and $s$ as non-zero constants, with Ritt-Wu's characteristic method, the above equations can be reduced to the following triangular form.

$$
\begin{aligned}
& f_{2}=\left(y_{1}-v\right) x_{2}-u y_{1}+v x_{1}=0 \\
& f_{1}=w y_{1}-s x_{1}=0 \\
& f_{4}=s^{2}\left(s^{2}+w^{2}\right) x_{1}^{4}-2 w s^{2}(v s+w u) x_{1}^{3}+\left(v^{2}+u^{2}-d_{1}^{2}\right) w^{2} s^{2} x_{1}^{2}+2 d_{1}^{2} w^{3} v s x_{1}-d_{1}^{2} w^{4} v^{2}=0
\end{aligned}
$$

With Theorem 3.1, we can calculate a cubic equation from the quartic equation $f_{4}=$ 0 . Using factorization algorithms from [22], we can show that this cubic polynomial is irreducible. This means that the diagram cannot be drawn with ruler and compass.

Example 3.3 (Pappus' Problem II) All requirements are the same as in Example 3.2 with an additional condition that point $P$ is on the bisector of angle $A O B$.

We need only to add a new constraint $\angle A O P=\angle P O B$ (with equation $d_{3}\left(v^{2}-u^{2}\right)+2 d_{2} u v=$ $0)$ to the constraint set of Example 3.2. Using Ritt-Wu's characteristic set method, we still get a quartic equation of $x_{1}$. This time the corresponding cubic equation is reducible and has the following linear factor of variable $g$

$$
-12\left(u^{2}+v^{2}\right)^{2} g+2\left(u^{2}-v^{2}\right)\left(u^{4}-v^{4}\right)+\left(u^{2}-v^{2}\right)^{2} d_{1}^{2}=0 .
$$


Then the quartic equation can be reduced to two quadratic equations and two linear equations as shown in Theorem 3.1 and the line can be drawn with ruler and compass.

Finally, let us comment that the above approach for quartic equations is similar to the well-known techniques of solving quartic equations. What we demonstrate here is that how this method can be obtained naturally in the context of solving rc-constructibility problems. Also, in the next section, we will use the same idea to solve the rc-constructibility problem in the general case.

3..3. The General Case. We want to know whether the roots of the following equation

$$
H(x)=x^{2^{n}}+h_{2^{n}-1} x^{2^{n}-1}+\ldots+h_{0}=0
$$

in $K[x]$ are rc-constructible. Let us first assume that the polynomial in (3.5) is irreducible which can be checked with the algorithm in [22].

If $K=\mathbf{Q}$, there exists a polynomial time algorithm to decide whether $H=0$ is solvable using radicals [17]. But in the general case, $K=\mathbf{Q}\left(u_{1}, \ldots, u_{q}\right)$. The method presented in [17] does not work in this case. One possibility is to give a probabilistic algorithm as follows. For a given polynomial $H$ involving parameters $u_{1}, \ldots, u_{q}$, we take $q$ random numbers $e_{1}, \ldots, e_{q}$ from $\mathbf{Q}$ and substitute $u_{i}$ for $e_{i}$ in $H$ to obtain a polynomial $H^{\prime} \in \mathbf{Q}[x]$. Then we can use the algorithm in [17] to decide whether the roots of $H^{\prime}=0$ are rc-constructible or equivalently whether they can be represented by square roots. If the roots of $H^{\prime}=0$ are not rc-constructible then the original problem is not rc-constructible. In the following, we will give a decision algorithm for the general case. However the algorithm has exponential time complexity.

Similar to the quartic case, if a root $y$ of equation (3.5) is rc-constructible, by (3.1) there is a number $x_{1}$ such that

$$
x_{1}=\frac{y^{2^{n-1}}+P(y)}{Q(y)} \text { and } x_{1}^{2}-c=0
$$

where $P$ and $Q$ are polynomials of degree $2^{n-1}-1$. Substituting $x_{1}$ into $x_{1}^{2}-c=0$, we have

$$
\left(y^{2^{n-1}}+P(y)\right)^{2}-c Q(y)^{2}=0 .
$$

Since an rc-constructible number is always a real number, we can assume $c>0$. Then the above equation becomes

$$
\left(y^{2^{n-1}}+P(y)\right)^{2}-(\sqrt{c} Q(y))^{2}=\left(y^{2^{n-1}}+P(y)\right)^{2}-R(y)^{2}=0 .
$$

Let $P=p_{2^{n}-1} x^{2^{n}-1}+\ldots+p_{0}$ and $R(y)=\sqrt{c} Q=q_{2^{n}-1} x^{2^{n}-1}+\ldots+q_{0}$. Substituting them into (3.6) and comparing coefficients with (3.5), we obtain a set of equations $P S$ about $p_{i}$ and $q_{i}$. It is clear that the solutions of $P S$ are in a one to one corresponding with the following factorization of $H$

$$
H(x)=\left(y^{2^{n-1}}+P(y)-R(y)\right) *\left(y^{2^{n-1}}+P(y)+R(y)\right)
$$

Lemma 3.4 Use the notations introduced above. The equation set PS has a finite number of solutions over any extension filed of $K$. 
Proof. Suppose that $H(x)$ has a factorization like

$$
H(x)=\left(y^{2^{n-1}}+s_{2^{n-1}-1} x^{2^{n-1}-1}+\ldots+s_{0}\right)\left(y^{2^{n-1}}+t_{2^{n-1}-1} x^{2^{n-1}-1}+\ldots+t_{0}\right) .
$$

Then by comparing the coefficients of equations (3.7) and (3.8), we have $p_{i}-q_{i}=t_{i}, p_{i}+q_{i}=s_{i}$ or $p_{i}-q_{i}=s_{i}, p_{i}+q_{i}=t_{i}$. In either case, we can determine $p_{i}$ and $q_{i}$ uniquely: $p_{i}=$ $\left(s_{i}+t_{i}\right) / 2, q_{i}=\left(s_{i}-t_{i}\right) / 2$ or $p_{i}=\left(s_{i}+t_{i}\right) / 2, q_{i}=\left(t_{i}-s_{i}\right) / 2$. Since polynomial $H$ can have only a finite number of factorizations like (3.8), there exist also a finite decompositions like (3.7). Since each set of solution of $P S$ will provide a decomposition (3.7), $P S$ has only a finite number of solutions over any extension field of $K$.

Now let us consider equation (3.6) more carefully. Let $k=2^{n-1}$. Considering the coefficients of $y^{2(k-1)}, y^{2 k-3}, \ldots, y^{k-1}$ in $R(y)^{2}$, the corresponding equations in $P S$ have the form

$$
\begin{aligned}
& R_{1}=q_{k-1}^{2}+f_{1}\left(p_{0}, \ldots, p_{k-1}\right)=0 \\
& R_{2}=2 q_{k-1} q_{k-2}+f_{2}\left(p_{0}, \ldots, p_{k-1}\right)=0 \\
& R_{3}=2 q_{k-1} q_{k-3}+f_{2}\left(p_{0}, \ldots, p_{k-1}, q_{k-2}\right)=0 \\
& \cdots \\
& R_{k}=2 q_{k-1} q_{0}+f_{k}\left(p_{0}, \ldots, p_{k-1}, q_{k-2}, \ldots, q_{1}\right)=0 .
\end{aligned}
$$

If $q_{k-1} \neq 0, R S=\left\{R 1, \ldots, R_{k}\right\}$ is in triangular form with leading variables $q_{k-1}, q_{k-2}, \ldots q_{0}$. Let $P$ be a polynomial in $P S$ but not in $R S$. If $P$ contains variables $q_{i}$, from equation (3.6) we see that $P=P_{1}\left(p_{0}, \ldots, p_{k-1}\right)+P_{2}\left(q_{0}, \ldots, q_{k-2}\right)$ and $P_{2}\left(q_{0}, \ldots, q_{k-2}\right)$ is a homogeneous quadratic polynomial. Since $q_{k-1} \neq 0, P=0$ is the same as $q_{k-1}^{2} P=0$. Using $R_{1}, \ldots, R_{k}$, we can eliminate all the $q_{i}$ from $P$. Let $P S_{1}$ be the set of polynomials obtained as above. We have reached the following result.

Lemma 3.5 Use the notations introduced above. If $q_{k-1} \neq 0$, the solutions of the equation set $P S$ can be obtained as follows. First we need to solve a polynomial equation $P S_{1}$ for the variables $p_{0}, \ldots, p_{k-1}$. Second $q_{0}, \ldots, q_{k-1}$ can be obtained with the triangular equations $R_{1}=0, \ldots, R_{k}=0 . P S_{1}$ also has a finite number of solutions.

We need only to show that $P S_{1}$ has a finite number of solutions. To prove this, we need only to show that each set of solutions of $P S_{1}$ will lead to a set of solutions for $P S$. For a set of solutions of $P S_{1}$, if $q_{k-1} \neq 0$ then we can obtain a set of of solutions for the $q_{i}$ via $R_{i}=0$. If $q_{k-1}=0$, let $l$ be the largest index such that $q_{l-1} \neq 0$. Then we can obtain a similar set of equations $R_{1}, \ldots, R_{l}$ from which the rest of the $q_{i}$ can be obtained.

Lemma 3.6 Use the notations introduced above. (1) Equation (3.5) has a decomposition like (3.6) iff $P S_{1}$ has a set of solutions in $K$.

(2) Let a set of solutions of $P S_{1}$ be $\left(e_{0}, \ldots, e_{k-1}\right), e_{i} \in K$. Then all roots $y$ of (3.5) can be obtained as follows

$$
\begin{aligned}
& y^{k}+e_{k-1} y^{k-1}+\ldots+e_{0}-\left(q_{k-1} y^{k-1}+\ldots+q_{0}\right)=0 \\
& R_{1}=q_{k-1}^{2}+f_{1}\left(p_{0}, \ldots, p_{k-1}\right)=0\left(q_{k-1} \neq 0\right) \\
& R_{2}=2 q_{k-1} q_{k-2}+f_{2}\left(e_{0}, \ldots, e_{k-1}\right)=0 \\
& R_{3}=2 q_{k-1} q_{k-3}+f_{2}\left(e_{0}, \ldots, e_{k-1}, q_{k-2}\right)=0 \\
& \cdots \\
& R_{k}=2 q_{k-1} q_{0}+f_{k}\left(e_{0}, \ldots, e_{k-1}, q_{k-2}, \ldots, q_{1}\right)=0 .
\end{aligned}
$$


If $q_{k-1}=0$, we can use the largest $l$ such that $q_{l} \neq 0$ as the leading coefficient for $R$ to find a similar set of equations.

Proof. The proof is similar to that of Theorem 3.1. For the first part, we already proved that each set of solutions of $P S_{1}$ can be extended to a set of solutions for $P S$. This means that each solutions of $P S_{1}$ will lead to a decomposition (3.6). Noting that in decomposition (3.6) $P(y)$ and $Q(y)$ are polynomials in $K[y]$. Then decomposition (3.6) exists iff $P S_{1}$ has a set of solutions in $K$.

For the second part, similar to the proof of Lemma 3.5, if $q_{k-1} \neq 0$ we can eliminate the variables $q_{i-2}, q_{i-3}, \ldots, q_{1}$ from $R_{i}$ for $i \geq 2$.

$$
\begin{aligned}
& R_{2}^{\prime}=2 q_{k-1} q_{k-2}+g_{2}\left(p_{0}, \ldots, p_{k-1}\right)=0 \\
& R_{3}^{\prime}=2 q_{k-1} q_{k-3}+g_{2}\left(p_{0}, \ldots, p_{k-1}\right)=0 \\
& \cdots \\
& R_{k}^{\prime}=2 q_{k-1} q_{0}+g_{k}\left(p_{0}, \ldots, p_{k-1}\right)=0 .
\end{aligned}
$$

Therefore, for any values of $p_{0}, \ldots, p_{k-1}, q_{k-1} q_{i}$ is a constant. When $q_{k-1}$ changes signs, $q_{i}(i=0, \ldots, k-2)$ will also change signs. Since $R_{1}=0$ will give raise to a positive and a negative values for $q_{k-1}$, choosing one of the two equations $y^{2^{n-1}}+P(y)-R(y)=0$ or $y^{2^{n-1}}+P(y)+R(y)=0$ is sufficient to find all the solutions of (3.5).

Before giving the algorithm, let us note that if a polynomial equation system $P S_{1}$ has a finite number of solutions over any extension field of $K$. Then the problem of deciding whether $P S_{1}$ has a set of solutions over $K$ is easy. This is equivalent to the fact that in a decomposition like (2.2) there exists a triangular set consisting of linear polynomials only.

Algorithm 3.7 The algorithm will decide whether a geometric constraint system is rcconstructible, and if it is, represents the coordinates of the points in the geometric problems as solutions of linear and quadratic equations.

Step 1. Choose a coordinate system. Let $P S$ be the algebraic equations of the geometric constraints.

Step 2. Using Ritt-Wu's decomposition algorithm, we can represent the solutions of $P S$ as solutions of polynomial sets in triangular form.

$$
\operatorname{Zero}(P S)=\bigcup_{i} Z \operatorname{ero}\left(T S_{i} / I_{i}\right)
$$

where $I_{i}$ is the product of the initials of the polynomials in $T S_{i}$.

Step 3. Let $T S_{i}=\left\{p_{1}\left(U, x_{1}\right), p_{2}\left(U, x_{1}, x_{2}\right), \ldots, p_{k}\left(U, x_{1}, \ldots, x_{k}\right)\right\}$ where $U=u_{1}, \ldots, u_{m}$ are the segment length and angle value parameters. If all the $p_{i}$ are linear and quadratic, then the diagram defined by $T S_{i}$ is rc-constructible. Otherwise, by (2.3), $\operatorname{Zero}\left(T S_{i} / I_{i}\right)$ can be represented in the following form ${ }^{6}$ )

$$
R_{i}(U, w)=0, x_{j}=M_{j}(U, w) / N_{j}(U, w),(j=1, \ldots, k)
$$

where $w$ is a new variable and $R, M_{i}, N_{i}$, are polynomials in $K[U, w]$.

\footnotetext{
${ }^{6)}$ A more efficient way is to allow quadratic equations of $x_{i}$ and we need only to change those $p_{i}$ with degree higher than two. Now, $Z \operatorname{ero}\left(T S_{i} / I_{i}\right)$ can be represented as $R_{i}(U, w)=0, Q_{j}\left(U, w, x_{j}\right)=0,(j=1, \ldots, k)$ where $Q_{j}$ is either a linear or a quadratic polynomial in $x_{j}$.
} 
Step 4. Now it is clear that the constraint system is rc-constructible iff the solution of each $R_{i}(U, w)$ is rc-constructible. We assume that $R_{i}(U, w)$ is irreducible, since otherwise we can factor it with the efficient algorithms in [22]. If the degree of $R_{i}$ in $w$ is not a power of two, the roots of $R_{i}$ are not rc-constructible. If it is, do Step 5 .

Step 5. Let $R_{i}$ be of degree $2^{m}$. Using Lemma 3.6, we can decide whether the roots of $R_{i}=0$ can be written as the roots of a set of linear and quadratic equations and the roots of an equation of degree $2^{m-1}$. Using this process repeatedly, we can decide whether the roots of $R_{i}$ can be written as the solutions of a set of linear and quadratic equations. If the answer is positive, the geometric problem is rc-constructible; otherwise, the geometric problem is not rc-constructible.

I

Although this decision procedure is an extension of the efficient algorithm for quartic equations, it is not efficient. This is because, in the case of quartic equations, the rcconstructibility is reduced to the reducibility of a cubic equation. For higher degree polynomials, the rc-constructibility is reduced to the solution of a set of quadratic equations $P S_{1}$. It is known that solving zero dimensional equations is single exponential in the number of variables [18]. Then our algorithm should also be of single exponential of the degree of the polynomial equation. Nevertheless, this seems to be first decision procedure for the rc-constructibility problem. Also, in practice equations with degree greater than four are rarely encountered.

\section{References}

[1] B. Buchberger, Gröbner Bases: an Algorithmic Method in Polynomial Ideal Theory, Recent Trends in Multidimensional Systems theory (ed. N.K. Bose), D.Reidel Publ. Comp., 1985.

[2] B. W. Char, et al, Maple V, Springer-Verlag, 1991.

[3] S.C. Chou, Mechanical Geometry Theorem Proving, D.Reidel Publishing Company, Dordrecht, Netherlands, 1988.

[4] S. C. Chou \& X. S. Gao, Mechanical Formula Derivation in Elementary Geometries, Proc. ISSAC-90, ACM, New York, 1990, p. 265-270.

[5] G.E. Collins, Infallible Calculation of Polynomial Zeros to Specified Precision, Mathematical Software, p. 35-68, Academic Press, New York, 1977.

[6] G. E. Collins, Quantifier Elimination for Real Closed Fields by Cylindrical Algebraic Decomposition, Lect. Notes Comp. Sci. 33, 134-183, Springer-Verlag, 1975.

[7] S.D. Conte \& C. de Boor, Elementary Numerical Analysis, Prentice-Hall, 1976.

[8] X.S. Gao \& S.C. Chou, On the Dimension for Arbitrary Ascending Chains, Chinese Bull. of Scis., vol. 38, pp.396-399, 1993.

[9] X.S. Gao \& S.C. Chou On the Theory of Resolvents and Its Applications, MM-Research Preprints, No.6, Inst. of Systems Science, Academia Sinica, (submitted to J. of Symbolic Computation).

[10] X.S. Gao \& S.C. Chou, Solving Geometric Constraint Systems, Part I. A Global Propagation Approach, WSUCS-96-1, CS Dept, Wichita State University, 1996 (submitted).

[11] C. Hoffmann, Geometric Constraint Solving in $R^{2}$ and $R^{3}$, in "Computing in Euclidean Geometry", eds D. Z. Du and F. Huang, p. 266-298, World Scientific, 1995.

[12] C. Hoffmann and I. Fudos, Constraint-based Parametric Conics for CAD, Geometric Aided Design, vol 28, No. 2, p.91-100, 1996.

[13] N. Jacobson, Basic Algebra, Vol. 1, San Francisco, Freeman, 1985. 
[14] K. Kondo, Algebraic method for manipulation of dimensional relationships in geometric models, Geometric Aided Design, 24(3), p. 141-147, 1992.

[15] G. Kramer, Solving Geometric Constraint Systems, MIT Press, 1992.

[16] A. Heydon \& G. Nelson, The Juno-2 Constraint-Based Drawing Editor, SRC Research Report $131 a, 1994$

[17] S. Landau \& L. Miller, Solvability by Radicals Is in Polynomial Time, J. of Computer and System Sciences, 30, p.179-208, 1985.

[18] D. Lazard, Solving Zero-Dimensional Algebraic Systems, J. of Symbolic Computation, 13/2. 117-131.

[19] V.C. Lin, D.C. Gossard, \& R.A. Light, Variational Geometry In Computer-Aided Design, Computer Graphics, vol. 15, no. 3, 171-177, 1981.

[20] J. Owen, Algebraic Solution for Geometry from Dimensional Constraints, in ACM symp. Found. of Solid Modeling, p. 397-407, Austin TX, 1991.

[21] J. Owen, Constraints of Simple Geometry in Two and Three Dimensions, To appear in Inter. J. of Comp. Geometry and Its Applications.

[22] P. S. Wang, An Improved Multivariate Polynomial Factorization Algorithm, Math. Computation, 32, p.1215-1231.

[23] J. F. Ritt, Differential algebra, Amer. Math. Sco. Colloquium, (1950).

[24] W. Whiteley, The union of Matroids and the Rigidity of Framework, Siam J. Disc. Math., vol 1, 237, 1988.

[25] Wu Wen-tsün, Basic Principles of Mechanical Theorem Proving in Geometries, Volume I: Part of Elementary Geometries, Science Press, Beijing (in Chinese), 1984. English version, SpringerVerlag, 1993. 